\title{
Hiding in plain sight-unreported osteoporotic vertebral fractures
}

\author{
Bo Abrahamsen ${ }^{1,2,3}$ (D) \\ Received: 6 April 2020 / Accepted: 7 May 2020 / Published online: 27 May 2020 \\ (C) European Society of Radiology 2020
}

\section{Key Points}

- Reporting of vertebral fractures has important clinical consequences and can be improved by routinely performing sagittal reformating and by the use of a standard nomenclature and classification.

There is a general expectation among our patients that they and their referring physician will be made aware of any pathologies that are visible in a radiology exam. As patients, we would certainly be annoyed to find that actionable abnormalities in our CT scan had not made it to the radiology report or that they had done so with a terminology that did not accurately alert the recipient to the nature of the pathology.

Osteoporotic vertebral fractures were always a diagnosis that was likely not to get a mention, even if easily visible on a lateral chest $\mathrm{x}$-ray. One reason that is sometimes given is that the referral was not about the health of the patient's vertebrae and that really it would be of no real value to the patient to be burdened with an additional health concern. A statement of some value in the 1970s, in 2020 it makes as little sense as arguing that the management of a patient would not be changed by the accurate reporting of a myocardial infarction on an ECG. After all, these two situations have a lot in common when it comes to the benefits to the patient of accurate reporting. Starting daily pravastatin after an MI leads to a $24 \%$ reduction in the risk of new MI [1], an outcome that comes with an in-hospital mortality of 3-5\% at age 70-79. Starting osteoporosis treatment with a mere annual dose of zoledronic acid after an osteoporotic vertebral fracture reduces the risk of hip fracture, a condition that also has an in-hospital mortality of $3-5 \%$, by $42 \%$ [2]. However, patients can rely on the

Bo Abrahamsen

b.abrahamsen@physician.dk

1 Department of Medicine, Holbæk Hospital, DK-4300 Holbæk, Denmark

2 OPEN Data Explorative Network, Institute of Clinical Research, University of Southern Denmark, C, DK-5000 Odense, Denmark

3 NDORMS, University of Oxford, Windmill Road, Headington, Oxford OX3 7LD, UK former action to be taken but are often not given the opportunity of the latter because fractures escape reporting. Consequently, more than half of women presenting with hip fractures have pre-existing vertebral fractures [3] though very few have been clinically recognized or treated [4].

In this issue, a nationwide audit in the UK [5] examined the organizational radiological reporting infrastructure and reviewed in detail the reporting that had been done for representative samples of individual CT studies that included the thoracolumbar spine. The UK has a strong culture of quality assurance in national healthcare with auditing of clinical services including radiology. It has been known for some time that vertebral fractures often go unreported even at very prestigious teaching hospitals and that it makes an unexpectedly large difference if the radiologist is an MSK radiology specialist or not [6]. Furthermore, the UK is very much the European front runner in terms of setting up clear pathways and fracture liaison services to close the treatment gap for secondary prevention of osteoporotic fractures [7]. It is likely that if things are not going well in terms of acting on vertebral fractures in the UK, then they may be even worse elsewhere where auditing is less ingrained in the healthcare system.

The audit focused on men and women above the age of 70 because this age range would be particularly likely to have vertebral fractures. Audit standards had been agreed beforehand through consultation with relevant stakeholders including the professional societies the Royal College of Radiologists, the Royal College of Physicians and the patient organization, the Royal Osteoporosis Society. Reassuringly, of 6256 CT studies, almost $80 \%$ contained comments about the integrity of the bones. However, more than two-thirds of the 614 studies showing vertebral fractures referred to this finding by other terms than vertebral fracture. Though one may think the choice of words may not matter much, terms such as "loss of height," "deformity," or "wedging" are unlikely to result in the patient 
receiving appropriate assessment for osteoporosis by the referring physician. Certainly, fewer than $3 \%$ of primary reports explicitly recommended referral or further assessment where the audit standard was $100 \%$. Thus, while it is unusual for the skeleton not to be mentioned in the report, the terminology used and actions suggested lack stringency. These shortcomings in reporting will limit the opportunities for access to secondary fracture prevention for the patient.

More importantly, the audit provides helpful insights into how clinical performance can be improved. First, reporting was significantly worse in exams where a sagittal reformat of the CT scan had not been done. Including this in your standard operating practice will in itself lower the likelihood of vertebral fractures being missed. Second, the poor agreement between classes of diagnostic and grading criteria for vertebral fractures $[8,9]$ is frustrating to radiologists and clinicians alike and may contribute to a lower quality in reporting. The semiquantitative Genant classification [10] is familiar to most radiologists and since this was also used as an entry criterion in most secondary intervention trials for vertebral osteoporosis, it provides a consistent approach to identifying patients with vertebral fractures who are candidates for osteoporosis assessment. Third, there has to be a fast, simple, and intuitive way for the presence of vertebral fractures to be brought to the attention of the service responsible for osteoporosis assessments, be it a local fracture liaison service, the referring physician, or another clinical path that makes sense in the local healthcare context. These are all relatively small steps for a radiology service to take but they will translate into substantially better long-term health outcomes for our patients.

\section{Compliance with ethical standards}

Conflict of interest Institutional research grants UCB, Novartis, KyowaKirin UK. Consulting or speakers fees UCB, Kyowa-Kirin UK, Amgen, Eli-Lily.

\section{References}

1. Sacks FM, Pfeffer MA, Moye LA et al (1996) The effect of pravastatin on coronary events after myocardial infarction in patients with average cholesterol levels. N Engl J Med 335:1001-1009. https://doi.org/10.1056/NEJM199610033351401

2. Eastell R, Black DM, Boonen S et al (2009) Effect of once-yearly zoledronic acid five milligrams on fracture risk and change in femoral neck bone mineral density. J Clin Endocrinol Metab 94:32153225

3. Gonnelli S, Caffarelli C, Maggi S et al (2013) The assessment of vertebral fractures in elderly women with recent hip fractures: the BREAK Study. Osteoporos Int 24:1151-1159. https://doi.org/10. 1007/s00198-012-2119-2

4. Frederiksen A, Abrahamsen B, Johansen PB, Sørensen HA (2018) Danish, national cross-sectional observational study on the prevalence of prior major osteoporotic fractures in adults presenting with hip fracture-limitations and scope for fracture liaison services in prevention of hip fracture. Osteoporos Int 29:109-114. https://doi. org/10.1007/s00198-017-4247-1

5. Howlett DC, Drinkwater KJ, Mahmood N, Illes J, Griffin J, Javaid K (2020) Radiology reporting of osteoporotic vertebral fragility fractures on computed tomography studies: results of a UK national audit. Eur Radiol. https://doi.org/10.1007/s00330-020-06845-2

6. Mitchell RM, Jewell P, Javaid MK, McKean D, Ostlere SJ (2017) Reporting of vertebral fragility fractures: can radiologists help reduce the number of hip fractures? Arch Osteoporos 12:71. https:// doi.org/10.1007/s11657-017-0363-y

7. Mitchell $\mathrm{P}$, Åkesson $\mathrm{K}$, Chandran $\mathrm{M}$, Cooper $\mathrm{C}$, Ganda $\mathrm{K}$, Schneider M (2016) Implementation of Models of Care for secondary osteoporotic fracture prevention and orthogeriatric Models of Care for osteoporotic hip fracture. Best Pract Res Clin Rheumatol 30:536-558. https://doi.org/10.1016/j.berh.2016.09.008

8. Oei L, Koromani F, Breda SJ et al (2018) Osteoporotic vertebral fracture prevalence varies widely between qualitative and quantitative radiological assessment methods: the Rotterdam Study. J Bone Miner Res 33:560-568. https://doi.org/10.1002/jbmr.3220

9. Ferrar L, Jiang G, Armbrecht $\mathrm{G}$ et al (2007) Is short vertebral height always an osteoporotic fracture? The Osteoporosis and Ultrasound Study (OPUS). Bone. 41:5-12

10. Genant HK, Wu CY, van Kuijk C, Nevitt MC (1993) Vertebral fracture assessment using a semiquantitative technique. J Bone Miner Res 8:1137-1148. https://doi.org/10.1002/jbmr.5650080915

Publisher's note Springer Nature remains neutral with regard to jurisdictional claims in published maps and institutional affiliations. 